\title{
Phase Oscillator to Enhance Human Running Performance by Wearable Robot
}

\author{
Zihao Leng, Zhuo Wang*, Junhong Yang, Qing Yang, Jianzhong Shang \\ College of Mechatronic Engineering and Automation, National University of Defense Technology, Changsha, 410073 China
}

\begin{abstract}
The human body is a spring-mass-damper system that oscillates up and down while moving. Wearable robots can add energy to the person at an appropriate time to enhance human moving performance and reduce the metabolic cost of performing cyclic tasks simultaneously. We have developed a phase oscillator to add assisted power at resonance so the system attains suitable energy to overcome the vertical resistance. The oscillator is robust to disturbances and initial conditions and allows wearable robots to enhance running, reduce metabolic cost, and increase hop height. Results show that when the external force is angled at 25 degrees, the phase oscillator can maintain robust bounded energy in a limit cycle to enhance human running performance.
\end{abstract}

\section{Introduction}

Wearable robotic systems can enhance and assist a user in a variety of tasks such as walking, running, and hopping [1]. However, achieving high running speeds is quite difficult because a large horizontal ground reaction force is needed to propel the person forward. For a human to run and support their body weight, they must utilize some of their largest muscles in the body which expend ample energy [2].

Human gait is a cyclical pattern of leg and foot movement that creates locomotion. The gait cycle can be divided into stance phase and swing phase. Stance phase begins with heel strike and ends when the toe of the same foot leaves the ground. Swing phase begins when the foot leaves contact with the ground and ends with heel strike of the same foot. Energy or metabolic expenditure during human gait depends on several factors including, body mass, stride length, step rate, and other physical and environmental factors. Other forces hindering human gait are air drag and rolling friction. Decreasing the metabolic cost for gaits allows the human to run faster or travel for a longer distance while minimizing the energy expended.

Human body can be described as a mass-springdamper system. Adding energy to the system at proper moment enhances oscillations of the human body during gait cycles. A small addition of energy results in a large resonance response. In this paper, we developed a phase oscillator to add assisted power at resonance so the system attains the suitable energy to overcome the vertical resistance. The oscillator is robust to disturbances and initial conditions and allows wearable robots and robots to enhance running, reduce metabolic cost, and increase hop height. Results show that when the external force is angled at 25 degrees, the phase oscillator can maintain robust bounded energy in a limit cycle to enhance human running performance.

\section{Phase Oscillator Design}

A general equation for a mechanical system is described by a second order system with a given mass, $m$, dissipating term, $b$, and stiffness, $k$.

$$
m \ddot{x}+b \dot{x}+k x=0
$$

A phase oscillator was developed to cancel the damping of the system to create a limit cycle based on the phase diagram. The controller adds energy to the system based on the phase angle, $\emptyset$, shown in Fig.1. [3].

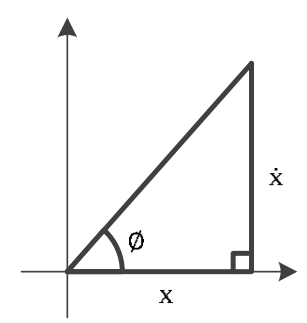

Figure 1. Phase plot definition, horizontal axis is the position $x$, and the vertical axis is the velocity $\dot{x}$. The sine of the phase angle $\varnothing$ is given by the opposite over the hypotenuse. The hypotenuse is given by $\sqrt{\dot{\mathrm{x}}^{2}+\mathrm{x}^{2}}$.

In order to control uncertain dynamics, a forcing function proportional to the sine of the phase angle can be used to add energy to the system.

$$
m \ddot{x}+b \dot{x}+k x=c \sin (\emptyset)=\frac{c \dot{x}}{\sqrt{\dot{x}^{2}+x^{2}}}
$$

In order to verify the feasibility of the phase oscillator control method, we set up a simulation model based on spring-mass-damper system, shown in Fig.2. 


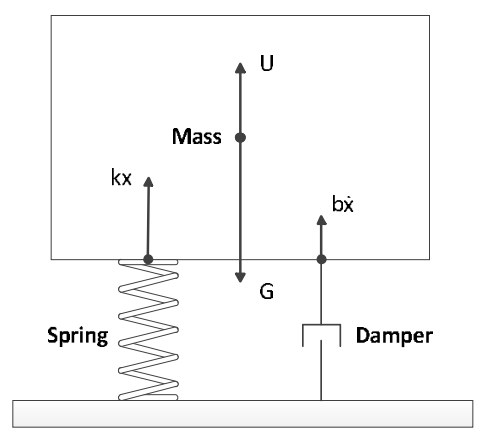

Figure 2. Spring mass damper simulating model.

The model was regarded as a mass with the quality of $\mathrm{m}$. The mass is connected with ground by the spring and damper. Mass moves up and down by the external force $U$. A phase angle $\varnothing$ of mass is used to determine the external force $U$.

To verify the robustness of the system, Eqs (1) and (2) were simulated in the software MATLAB/Simulink, shown in Fig. 3. $M=1 \mathrm{~kg}, \mathrm{~b}=0.6 \mathrm{~N} /(\mathrm{m} / \mathrm{s}), \mathrm{k}=200 \mathrm{~N} / \mathrm{m}$, initial velocity $=1.2 \mathrm{~m} / \mathrm{s}$, and initial position $=0 \mathrm{~m}$ [4]

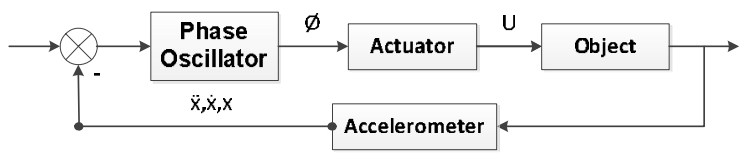

Figure 3. The phase oscillator control flow diagram

The negative damping system modelled in Eqs (1) without phase oscillating system damps to zero. The system modelled in Eqs (2) with phase oscillating system position oscillates up and down. If $\mathrm{c}$ is negative, the system is damped, and because of the negative energy the system state goes to zero faster than the first simulation. If $\mathrm{c}$ is positive, the system oscillates up and down. The energy is always bounded because the forcing function must be between plus or minus c, shown in Fig. 4 and 5.

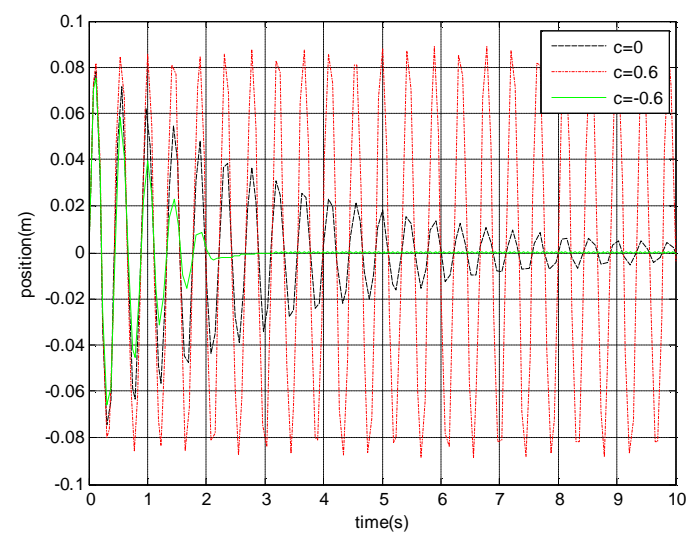

Figure 4. $c=0$. The negative damping system modelled in Eqs (1) damps to zero. $\mathrm{c}=-0.6$. The negative damping system modelled in Eqs (1) damps to zero. $\mathrm{c}=0.6$. The negative damping system modelled in Eqs (2) up and down.

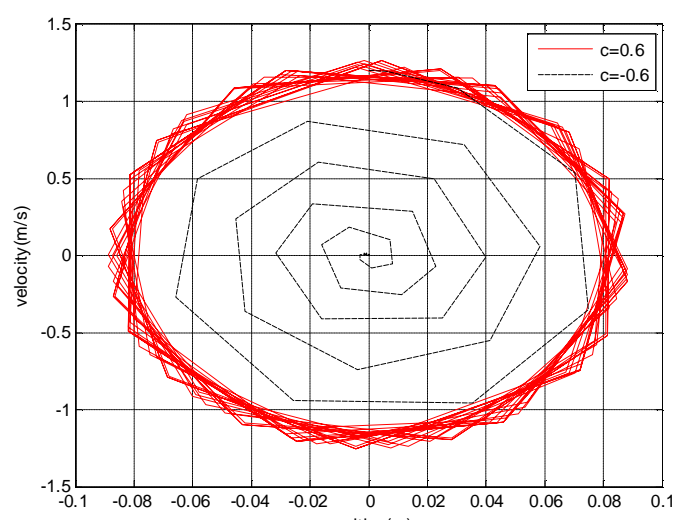

Figure 5. $c=-0.6$. The system damps to zero. $c=0.6$. The system is bounded in a cycle.

\section{Running Performance Simulation Based on Phase Oscillator}

In the vertical plane, the main force hindering the running motion is gravity. In the horizontal plane, the main forces hindering the running motion are air drag and rolling friction, shown in Fig.6.

The drag force multiplied by the runner's speed is the amount of power that hinders the runner's motion. Density $=1.225 \mathrm{~kg} / \mathrm{m}^{3}, A=0.55 \mathrm{~m}^{2}, C=1.36$, and $V$ is the velocity of the runner.

$$
P_{d r a g}=0.5 \rho A C V^{3}
$$

The rolling friction between the ground and the shoe also creates a power that hinders the runner's motion. Mass $=80 \mathrm{~kg}$, rolling friction constant, $A_{\mathrm{r}}=0.02$, and $V$ is the velocity of the runner. We use three times the body weight because the runner is hitting the ground quite hard [2].

$$
P_{\text {friction }}=3 m g A_{r} V
$$

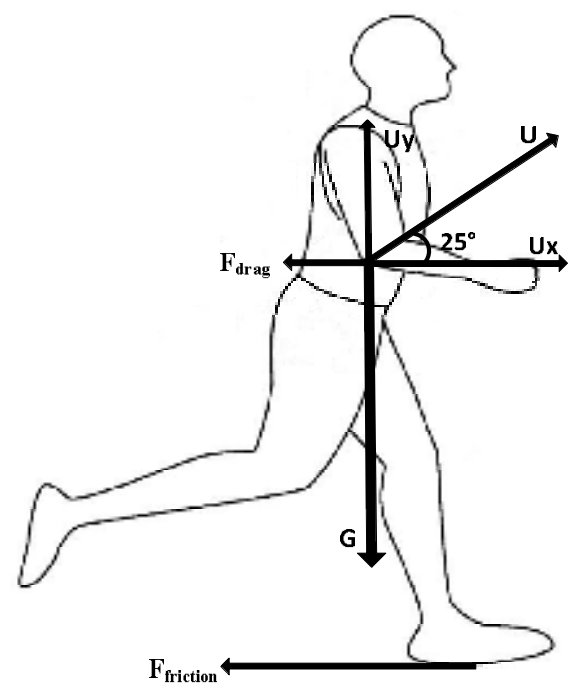

Figure 6. Forces acting on the runner.

To enhance the motion of human motion during gait, phase oscillating system adds a parametric excitation 
force, external force $U$, to produce positive power for human body.

It was determined that the external force $U$ should be angled at 25 degrees so that an upward force as well as a propulsive force is generated. Both vertical and horizontal forces aid human running [2].

The horizontal force $U_{\mathrm{x}}$ was determined by the horizontal velocity $6.7 \mathrm{~m} / \mathrm{s}$ for running motion.

The direction of external force $U_{\mathrm{y}}$ is switched at a time and frequency that is tuned in phase with the frequency of the gait step. The external force $U_{\mathrm{y}}$ changes its direction based on human body motion, shown in Fig.7.

Quadrant 1 represents the toe off phase of gait where human body has a positive velocity, human body is moving upwards in the air. To add positive power to the toe off phase for human body, phase oscillating system provides a positive external force $U_{\mathrm{y}}$.

Quadrant 2 represents the phase of gait where human body has a negative velocity, human body is falling back towards the ground. In order to add positive power to the falling phase for human body, phase oscillating system provides a negative or downward external force $U_{\mathrm{y}}$ to drive human body towards the ground.

Quadrant 3 represents the phase of landing where human body continues with a negative velocity toward the ground. In order to add positive power, phase oscillating system provides a negative or downward external force $U_{\mathrm{y}}$ to further compress the legs, similar to compressing a spring.

Quadrant 4 represents the phase of human extends the legs and pushes off the ground. In order to add positive power to the push off phase of gait for human body, phase oscillating system provides a positive external force $U_{\mathrm{y}}$.

According to the force analysis, we made a phase oscillator based on the human body with gravity. The human body can be described as a structure with mass, damping, and spring stiffness. The leg stiffness and damping are only active when the human hits the ground and the leg is compressed. The external force $U_{\mathrm{y}}$ allows the system to oscillate up and down based on sine of the phase angle.

$$
m \ddot{y}+b \dot{y}+k y+m g=c \sin (\emptyset)=\frac{c \dot{y}}{\sqrt{\dot{y}^{2}+y^{2}}}
$$

In order to verify the feasibility of the phase oscillator control method and determine suitable external force $U$, we set up an MATLAB/Simulink model. $M=80 \mathrm{~kg}, \mathrm{~b}=$ $4 \mathrm{~N} /(\mathrm{m} / \mathrm{s}), \mathrm{k}=160000 \mathrm{~N} / \mathrm{m}$, initial velocity $=1.2 \mathrm{~m} / \mathrm{s}$, and initial position $=0 \mathrm{~m}$.

According to the Fig.7, when $\mathrm{c}=0.8$, the limit cycle is robust. The system oscillates up and down with good stability. The damping of the leg creates resistive force to slow the system down while the external force created by the sine of the phase angle assists the movement of the system. The external forces are shown in Fig.8.

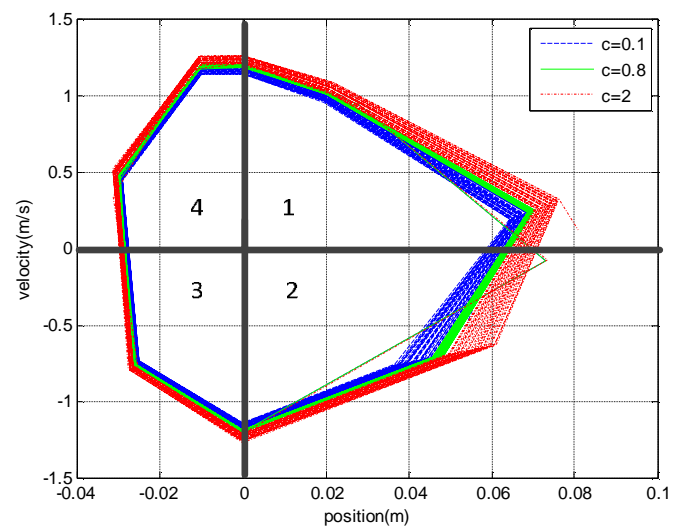

Figure 7. $\mathrm{c}=0.8$. The system maintains a stable limit cycle. $\mathrm{c}=$ 0.1 . The system damps out of the limit cycle. $\mathrm{c}=2$. The system damps inside the limit cycle.

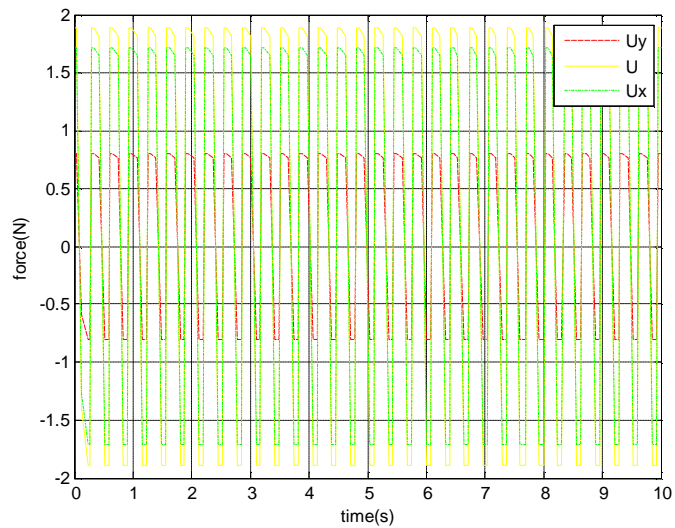

Figure 8. The control forces are continuous and are generated by the sine of the phase angle.

To hinder or dampen the hopping motion of human body during gait, phase oscillating system provides a negative power. The direction of external force $U$ is switched at a time and frequency that is tuned in antiphase with the frequency of the gait step. To subtract or absorb energy to the toe off phase for human body, phase oscillating system provides a positive external force $U$ in the landing phase or the push off phase, provides a negative external force $U$ in the toe off phase or the falling back phase.

\section{Conclusions}

Our goal has been to develop a method to assist the human motion while running. We developed a phase oscillator to develop a control signal that is bounded and creates a limit cycle. The control signal is continuous and is used to trigger the wearable robot to develop the external force. The results of the simulation show that when the external force is angled at 25 degrees, the phase 
oscillator can maintain robust bounded energy in a limit cycle to enhance human running performance.

\section{Acknowledgments}

This work performed under the auspices of National University of Defence Technology Project (JC14-03-03), Natural Science Foundation of Hunan Province (2015JJ3021), and National Natural Science Foundation of China (60908022, 51205400).

\section{References}

[1] T. G. Sugar, A. Bates, M. Holgate, J. Kerestes, M. Mignolet, P. New, R. K. Ramachandran, S. Redkar, and C. Wheeler, "Limit Cycles to Enhance Human Performance Based on Phase Oscillators," Journal of Mechanisms and Robotics. Vol.7, No.011001, pp. $1-8(2015)$.

[2] J. Kerestes, and T. G. Sugar, "Enhanced Running Using A Jet Pack," IDETC. Vol.05, No.08A006, pp. 1-7(2014).

[3] J. Kerestes, T. G. Sugar, T. Flaven, M. Holgate, and R. K. Ramachandran, "A Method to Add Energy to Running Gait - Pogo Suit," IDETC. Vol.05, No.08A005, pp. 1-6(2014).

[4] J. Kerestes, T. G. Sugar, and M. Holgate, "Adding and Subtracting Energy to Body Motion - Phase Oscillator," IDETC. Vol.05, No.08A004, pp. 16(2014) 